\title{
A CASE REPORT OF ISOLATED BRAINSTEM TUBERCULOMA WITHOUT ANY FOCAL NEUOLOGIC DEFICIT
}

\author{
T. V. Ramesh ${ }^{1}$, V. Rahul ${ }^{2}$ \\ 1Professor, Department of Paediatrics, GSL Medical College, Rajamundry, Andhra Pradesh. \\ ${ }^{2}$ Resident, Department of Paediatrics, GSL Medical College, Rajamundry, Andhra Pradesh.
}

ABSTRACT: A 7 yr. old girl presented with headache and vomitings. Investigations showed brainstem tuberculoma at the level of pons. Isolated presentation of tuberculoma of braistem is rare. In this report, it was aimed to present isolated brainstem tuberculoma without any focal neulogic deficit and other systemic involvement or past or family history of tuberculosis and importance of neuroimaging in diagnosing tuberculoma even in the absence of neulogic defcit.

KEYWORDS: Tuberculoma, Brainstem.

HOW TO CITE THIS ARTICLE: T. V. Ramesh,V. Rahul. "A Case Report of Isolated Brainstem Tuberculoma without any Focal Neuologic Deficit”. Journal of Evolution of Medical and Dental Sciences 2015; Vol. 4, Issue 90, November 09; Page: 15601-15602, DOI: $10.14260 /$ jemds/2015/2240.

INTRODUCTION: Tuberculosis is still endemic in developing countries. Neurotuberculosis is significantly contributing the mortality and morbidity in pediatric patients. Tubercular meningitis and intracranial tuberculomas are the two frequent manifestations of neurotuberculosis. A tuberculoma is a tuberculous focus, which results from aggregation of caseous tubercles. Tuberculoma usually manifest raised intracranial manifestations and focal neurologic deficit simulates brain tumour. CNS Tuberculomas, either multiple or single, usually presents as a diagnostic challenge, specifically in patients without constitutional symptoms or evidence of tuberculosis elsewhere in the body.

CASE REPORT: A 7 yr. old female child presented with complaints of headache since 3 days, vomitings since 3 days. Severe headache all over the head. Vomitings are present and are non-bilious non-projectile. Developmental history normal appropriate for the age. Immunization history followed national immunization schedule. BCG scar present. No past history of tuberculosis or other significant history. No family h/o tuberculosis. On general examination child is concious, irritable. Vitals are normal. No cranial nerve palsy. On motor system examination power, bulk, tone normal no focal neurologic deficit. No involuntary movements. No sensory loss. No autonomic distubances. No signs of menigeal irritation. Skull and spine normal. Gait normal. On MRI scan there is solitary tubercular granuloma present at level of pons. Fundoscopy normal, no optic disc edema. The TST is reactive $(12 \mathrm{~mm})$. Chest $\mathrm{X}$ Ray is normal.

Financial or Other, Competing Interest: None.

Submission 19-10-2015, Peer Review 20-10-2015,

Acceptance 28-10-2015, Published 09-11-2015.

Corresponding Author:

Dr. T. V. Ramesh

Flat No: F1, Satyasri Nilayam,

D. No: 65-4-6/A, Mehar Nagar,

Kakinada-533002, Andhra Pradesh.

E-mail: drtvr2010@gmail.com

DOI: $10.14260 /$ jemds $/ 2015 / 2240$.
DISCUSSION: In developing countries, the incidence of brain tuberculoma constitutes $5-40 \%$ of intracranial SOL. ${ }^{1}$ Lesions are most often singular but may be multiple. Isolated brainstem tuberculomas are rare, accounting for $2.5-8 \%$ of all intracranial tuberculomas. ${ }^{2}$ Isolated tuberculomas presenting as mass lesions in the brain stem without evidence of tuberculous involvement of any other system or other parts of the CNS, are distinctly rare $^{3}$ it has been reported that the predominant locations of tuberculoma are supratentorial in adults and infratentorial in children. ${ }^{4}$ the most common symptoms are headache, fever, focal neurologic findings, and convulsions. The TST is usually reactive, but the chest radiograph is usually normal. On CT or MRI of the brain, tuberculomas usually appear as discrete lesions with a significant amount of surrounding edema. Contrast medium enhancement is often impressive and can result in a ring-like lesion.

Vimla et al 15 reported a 12 year old patient, who presented with isolated "11/2 syndrome". 5 Piyush Gautam et al reported Isolated Pontine Tuberculoma Presenting as Horizontal Gaze Palsy. ${ }^{6}$ In our case there no neurological deficit except severe headache and vomitings. Important and clinically interesting and significant observation is there is an evidence of paradoxic expansion the lesions may occur some times and may lead to worsening the clinical condition. ${ }^{7}$ This paradoxic response usually occurs during first 3 months from the start of therapy. ${ }^{8}$ Corticosteroids therapy should be given and ATT should be continued.

CONCLUSION: Isolated brainstem tuberculosis is very rare presentation. Important thing to be remember is there is chance of paradoxic enlargement of the lesion or may be appearance of new lesions may occur due to enhancement of immuneresponse during the treatment. And it is not an indication of stopping treatment.

\section{REFERENCES}

1. Shah GV. Central Nervous System tuberculosis imaging manifestations. Neuroimaging Clin N Am 2000; 10: 355374.

2. Kumar R, Jain R, Kaur A, Chhabra DK. Brain stem tuberculosis in children. Br J Neurosurg. 2000;14: 356-61. 
3. Rajshekhar V, Chandy MJ. Tuberculomas presenting as isolated intrinsic brain stem masses. Br J Neurosurg 1997; 11: 127-133.

4. Traub M. Tuberculosis of the central nervous system. Clinical Neurology 1991; Vol 1: 875-877.

5. Vimla M, Madhurjya G, Rohit S, Sumit S, Arvind K. Isolated "One and a half syndrome" with brain stem tuberculoma. Indian J Pediatr 2004; 71: 469-471.

6. Piyush Gautam and Nivedita Sharma ;Department of Pediatrics, Dr R. P. Medical College and Hospital, Tanda, Kangra, Himachal Pradesh, India : Indian Pediatr 2015;52: 166.
7. Hejazi N, Hassler W. Multiple intracranial tuberculomas with atypical response to tuberculostatic chemotherapy: literature review and a case report. Acta Neurochir (Wien) 1997;139:194-202.

8. Wong GW, Oppenheimer SJ, Poon W, Leung R. Intracranial tuberculoma and hydrocephalus developing during treatment of tuberculous meningitis. J Trop Med Hyg 1994; 97(4): 236-238.

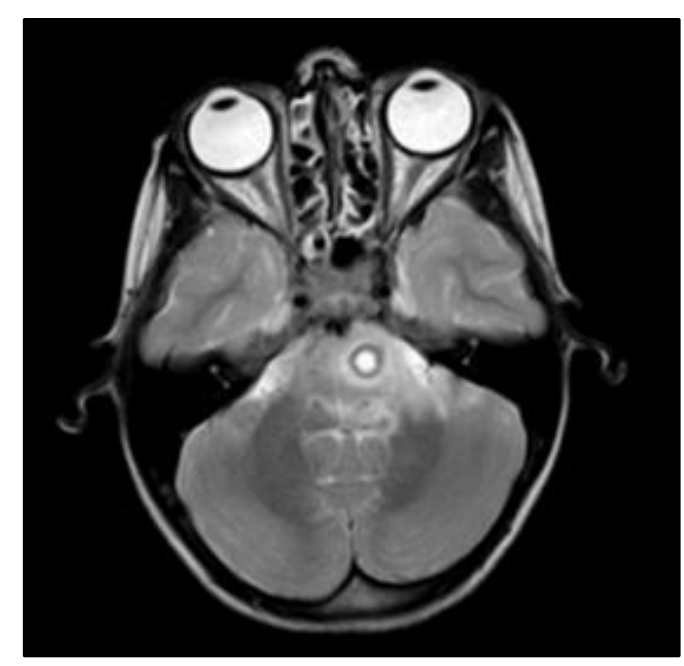

MRI axial section showing tuberculoma at the level of pons

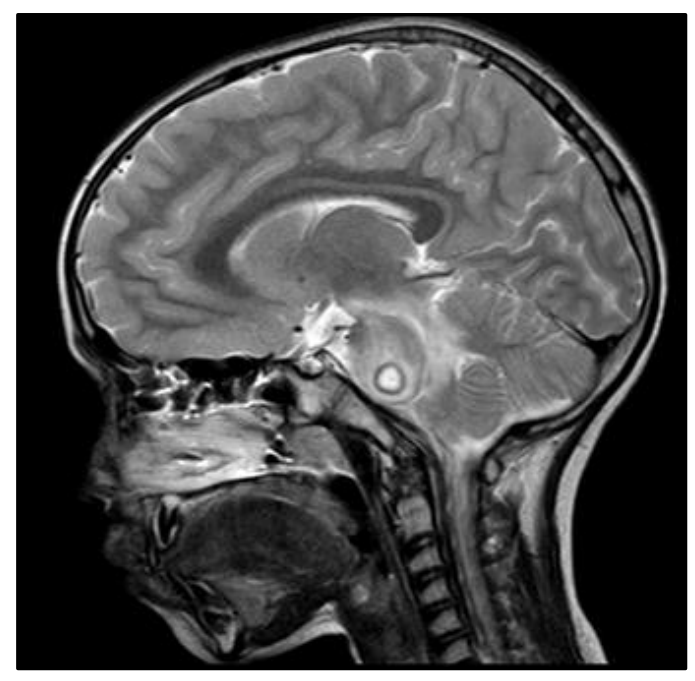

MRI sagittal section showing tuberculoma at the level of pons 